\title{
Occurrence and Detection of AmpC $\beta$-Lactamases among Enterobacteriaceae in a Tertiary Care Centre in Trivandrum, India
}

\author{
Ashish Jitendranath ${ }^{1 *}$, Vishnupriya Anoobis ${ }^{2}$, Geetha Bhai ${ }^{1}$, \\ Ivy Vishwamohanan ${ }^{1}$ and J.T. Ramani Bai ${ }^{3}$ \\ ${ }^{1}$ Department of Microbiology, Sree Gokulam Medical College and Research Foundation, India \\ ${ }^{2}$ Biosytech medical lab, Dubai \\ ${ }^{3}$ Department of Microbiology, S R Medical College, India \\ *Corresponding author
}

\section{A B S T R A C T}

\begin{tabular}{|c|c|}
\hline $\begin{array}{l}\text { K e y w o r d s } \\
\text { Enterobactericiae, Amp } \\
\text { C, E. coli, Boronic acid, } \\
\text { Imipenem, Meropenem }\end{array}$ & $\begin{array}{l}\text { The prevalence of multi drug resistant gram negative bacteria has increased continuously } \\
\text { over the past few years, of particular concern are strains producing AmpC } \beta \text {-lactamases. } \\
\text { The irrational uses of antibiotics are the primary cause of the increase in drug resistant } \\
\text { bacteria. Several studies have reported that AmpC producers are isolated mainly from }\end{array}$ \\
\hline Article Info & \\
\hline $\begin{array}{l}\text { Accepted: } \\
\text { 04 July } 2018 \\
\text { Available Online: } \\
10 \text { August } 2018\end{array}$ & $\begin{array}{l}\text { indicate their presence in the community. All the organisms were identified according to } \\
\text { standard methods. They were confirmed with Vitek } 2 \text {. E. coli and Klebsiella spp were the } \\
\text { most common gram negative organism while AmpC producers were found to be } 11.2 \% \text { of } \\
\text { the isolated Enterobactericiae }\end{array}$ \\
\hline
\end{tabular}

\section{Introduction}

The prevalence of multi drug resistant gram negative bacteria has increased continuously over the past few years, of particular concern are strains producing AmpC $\beta$-lactamases (Soha, 2015; Jacoby, 2009).

The irrational uses of antibiotics are the primary cause of the increase in drug resistant bacteria. There is no doubt that the misuse of the drug in human beings has contributed to the increasing rates of the resistance, but recently the use of antibiotics in veterinary animals and agriculture and its consequent effects on resistance levels in peoples has also come under scrutiny (Randall, 2003). Several studies have reported that AmpC producers are isolated mainly from patients with prolonged hospitalization, but certain recent studies have reported that AmpC producers was also isolated from long term care facilities and outpatient clinics, which indicate their presence in the community (Arindam, 2015) AmpC $\beta$-lactamases are clinically significant because they may confer resistance to penicillins, cephalosporins, oxyiminocephalosporins, cephamycins and monobactams (Soha, 2015; Polsfuss, 2005). 
A high rate of clinical failure among patients who were infected with AmpC $\beta$-lactamases producing bacteria and who received initial antimicrobial therapy, especially cephalosporin has been demonstrated. Therefore, detection of AmpC producing organisms is important to ensure effective therapeutic intervention and optimal clinical outcome (Tan, 2009).

There are no Clinical Laboratory Standards Institute (CLSI) or other approved criteria for AmpC detection. The detection of AmpC $\beta-$ lactamases is a challenge for routine clinical microbiology laboratories in resource limited settings (Mona Waseef, 2013). An initial screen for reduced susceptibility to more than one of the five indicator cephalosporins followed by a confirmatory test can improve the sensitivity of detection. The further identification of specific genes associated with the production AmpC $\beta$-lactamases can be performed using specific nucleic acid-based assays. (Gupta, 2007). However these molecular assays are limited to resource-rich settings and are beyond the scope of routine microbiology laboratories. (Anandkumar Harwalkar, 2013)

This study aimed to find out the characterization of Enterobacteriaceae and antibiotic sensitivity patterns with special reference to detection of AmpC $\beta$-lactamases producing Enterobacteriaceae in patients in a tertiary care hospital in south Kerala.

\section{Materials and Methods}

Present study was conducted in the Microbiology laboratory of Sree Gokulam Medical College and Research Foundation over a duration of 3 months from Oct 2015 to Dec 2015.

All the samples received in bacteriology section of laboratory were inoculated on blood agar, MacConkey agar and incubated at $37^{\circ} \mathrm{C}$ for $48 \mathrm{~h}$ before being reported as sterile.

The isolates were identified based up on Colony characteristics, Gram staining, Motility and Conventional biochemical test like oxidase test, catalase test, indole, methyl red, Voges-Proskauer, citrate utilization, urease production oxidative fermentative test (Hugh-Leifson medium) for glucose, utilization of $10 \%$ lactose, gelatinliquefaction, lysine and ornithine decarboxylation, arginine dihydrolase test, growth at $42^{\circ} \mathrm{C}$ and $44^{\circ} \mathrm{C}$, esculin hydrolysisand ONPG test (Win Washington, 2014).

The sensitivity test was performed by Kirby bauer disc diffusion method using commercially available discs (Himedia). The results were interpreted as per the CLSI guideline.

\section{AmpC $\beta$-lactamases detection}

\section{Screening method for AmpC $\beta$-lactamases}

Organisms showing resistance to Cefoxitin (zone size $<18 \mathrm{~mm}$ ) should be considered as probable AmpC producer and should be confirmed by other methods (Supriya Upadhyay, 2011)

\section{Modified double disc approximation test}

Ceftazidime $(30 \mu \mathrm{g})$, Cefotaxime $(30 \mu \mathrm{g})$, are placed at a distance of $20 \mathrm{~mm}$ from Cefoxitin $(30 \mu \mathrm{g})$ on a MHA plate inoculated test organisms as per CLSI.

Isolates showing blunting of Ceftazidime or Cefotaxime zone of inhibition adjacent to Cefoxitin disc or showing reduced susceptibility to either of the above drug and Cefoxitin are considered as screening positive. (Hemalatha, 2007). 


\section{Detection of AmpC by boronic acid disc test}

Two types of Boronic acid discs can be used; one is commercially available with $250 \mu \mathrm{g}$ concentration and the other is home made with $400 \mu \mathrm{g}$ concentration (Mona Waseef, 2013).

\section{Boronic acid disc preparation}

Dissolve $120 \mathrm{mg}$ of Phenyl Boronic Acid were added to $3 \mathrm{ml}$ of Dimethyl Sulfoxide (DMSO). Then 3milliliters of sterile distilled water were added. Twenty micro-litters of the stock solution were dispensed on to discs containing $30 \mu \mathrm{g}$ of Cefoxitin. Allow the disc to air dry for 60 minutes and store in an air tight container at $4^{\circ} \mathrm{C}$ (Pai, 2004).

\section{Combined disc method}

Test organisms are inoculated to MHA as per CLSI. A disc containing 30 $\mu \mathrm{g}$ of Cefoxitin and other $30 \mu \mathrm{g}$ of Cefoxitin impregnated with $400 \mu \mathrm{g}$ Boronic acid are placed on MHA plate and are incubated at $35^{\circ} \mathrm{C}$ over night. An organism with a zone diameter of $\geq 5 \mathrm{~mm}$ around the disc containing Cefoxitin + Boronic acid, than the zone diameter around Cefoxitin disc alone is consider as a positive test (Mona Waseef, 2013).

\section{Results and Discussion}

From October 2017 to January 2018, total 250 consecutive non-repetitive Enterobacteriaceae isolates were tested for detecting their antimicrobial susceptibility pattern and production of AmpC beta-lactamases by a screening and confirmatory testing.

The source of the isolates was from urine, sputum, pus, blood and CSF. The clinical spectrum represents out of 250 isolates, 145 are isolated from urine, 57 from pus, 45 from sputum, 2 from blood and 1 from CSF. Distribution pattern of isolated organisms shows that $E$. coli was the most commonly isolated organism followed by Klebsiella. Out of 250, 134 isolates were E. coli, after that Klebsiella, which were about 80 out of 250 . Then Proteus (11), Citrobacter (10), Enterobacter (8), Serratia (4) and Morganella (3) respectively.

Overall antibiotic resistance pattern of the isolates showed high degree of resistance towards Ampicillin (86\%), Piperacillin (80\%), Amoxyclav (70\%) and third generation Cephalosporins (50\%). This was statistically significant with $\mathrm{p}$ value $<0.001$. Imipenam (6\%), Meropenam (6\%), Colistin (0\%), Cefoperazone/Sulbactam (14\%), Piperacillin/ Tazobactum (10\%) and Amikacin (10\%) had lower level of resistance

Drug resistance causes a therapeutic problem not only in the hospital settings, but also in the community as most of the bacteria have acquired resistance to multiple antibiotics. Various drug resistance mechanisms of Enterobacteriaceae include extended spectrum $\beta$-lactamases (ESBL) production, AmpC $\beta$ lactamases production, efflux mechanism, plasmids and porin deficiency. In clinical laboratory settings, commonly the commonly detected enzymes causing resistance are ESBLs and AmpC $\beta$-lactamases. Clinical relevance of AmpC $\beta$-lactamases lies in the fact that they confer resistance to both narrow and broad spectrum cephalosporins, $\beta$-lactam / $\beta$-lactamase inhibitor combinations and Astreonam.

Detection of AmpC $\beta$-lactamases from Enterobacteriaceae are important to improve the clinical management of patients suffering from infections and would also provide us with sound epidemiological data. However laboratory methods for screening and confirmation of ESBL should be accurate, simple and rapid. Whereas there are no Clinical and Laboratory Standard Institute 
(CLSI) guidelines for detection of AmpC $\beta$ lactamase

In the present study, out of 250 isolates studied, 134(54\%) are E. coli, and are the predominant isolates, followed by Klebsiella spp which are about $80(32 \%)$ cases and 8(3.2\%) cases of Enterobacter and 10(4\%) cases of Citrobacter were obtained. This result is comparable with a similar study conducted by Polsfuss et al., (2005) in their study, out of 2518 samples, 1435(57\%) were E.coli, 459(18.2\%) were Klebsiella, 178(5.2\%) were Enterobacter and $1(0.1 \%)$ case of Citrobacter were isolated (Polsfuss, 2005). But in the study conducted by Tapan Majumdar, Shibabrata Bhattacharya et al., they found out of 200 tested strains of Enterobacteriaceae, 50 cases were reported as E.coli, 40 cases of Klebsiella spp, 12 cases of Enterobacter and 8 cases of Citrobacter were obtained (Tapan Majumdar, 2014). But in the study of Leslie Jose Selvaraj et al., out of 993 Enterobacteriaceae Isolates, 510 cases were reported as E. coli, whereas 277 cases are Klebsiella spp and 168 cases are Enterobacter spp (Leslie Jose Selvaraj, 2008).
The present finding of antibiotic susceptibility pattern was out of 250 cases, antibiotic resistance were Ampicillin 85.4\%, Cefuroxime $60.8 \%$, Ceftazidime $52 \%$, Cefepime $36.4 \%$, Cefoxitin $31.6 \%$ and Imipenem 5\% only.

From the analysis, data obtained, bases on the antibiotic resistance pattern, the drugs such as Ampicillin, Cefuroxime, ceftazidime, cefepime and Imipenem shows a significant sensitivity pattern that is, $\mathrm{p}<.0001$

Similarly the Study of Tapan Majumdar et al., it was obtained that over all antibiotic resistant pattern of the isolates showed high degree of resistance towards Amoxycillin, $3^{\text {rd }}$ generation Cephalosporins and Tetracycline whereas Cefepime, Imipenam Aminoglycosides were showing less resistance (Tapan Majumdar, 2014). The study conducted by Anandkumar Harwalkar et al., revealed that out of 153 isolates, (93.8\%) was resistant to Ampicillin, 62(38\%) were resistant to Trimethoprim / Sulfamethoxazole (Anandkumar Harwalkar, 2013) (Table 1-3).

Table.1 Distribution of organisms in different samples

\begin{tabular}{|l|l|l|l|l|l|l|}
\hline $\begin{array}{l}\text { Organism } \\
(\mathbf{n = 2 5 0})\end{array}$ & Urine & Pus & Sputum & Blood & CSF & Total \\
\hline E.coli & 108 & 19 & 4 & 2 & 1 & 134 \\
\hline Klebsiella & 32 & 14 & 34 & 0 & 0 & 80 \\
\hline Enterobacter & 2 & 3 & 3 & 0 & 0 & 8 \\
\hline Proteus & 2 & 9 & 0 & 0 & 0 & 11 \\
\hline Citrobacter & 1 & 7 & 2 & 0 & 0 & 10 \\
\hline Serratia & 0 & 2 & 2 & 0 & 0 & 4 \\
\hline Morganella & 0 & 3 & 0 & 0 & 0 & 3 \\
\hline Total & 145 & 57 & 45 & 2 & 1 & 250 \\
\hline
\end{tabular}


Table.2 Susceptibility pattern of Enterobacteriaceae

\begin{tabular}{|l|l|l|}
\hline Antibiotics (N=100) & Resistant (\%) & Sensitive (\%) \\
\hline Ampicillin & 86 & 14 \\
\hline Piperacillin & 80 & 20 \\
\hline Amoxyclav & 70 & 30 \\
\hline Cephalexin & 69 & 31 \\
\hline Cefuroxime & 60 & 40 \\
\hline Cefotaxime & 52 & 48 \\
\hline Ceftaridime & 52 & 48 \\
\hline Cefepime & 36 & 64 \\
\hline Gentamicin & 32 & 67 \\
\hline Netilmicin & 15 & 85 \\
\hline Amikacin & 13 & 87 \\
\hline Ciprofloxacin & 42 & 58 \\
\hline Norfloxacin & 38 & 62 \\
\hline Ofloxacin & 42 & 58 \\
\hline Nitrofurantoin & 26 & 74 \\
\hline Cotrimoxazole & 36 & 64 \\
\hline Tetracycline & 41 & 59 \\
\hline Cefoperazone/sulbactum & 14 & 86 \\
\hline Piperacillin/Tazobactum & 12 & 88 \\
\hline Imipenem & 6 & 94 \\
\hline Meropenem & 6 & 94 \\
\hline Aztreonam & 41 & 59 \\
\hline Cefoxitin & 32 & 68 \\
\hline Colistin & 6 & 94 \\
\hline & & \\
\hline
\end{tabular}

Table.3 Distribution of AmpC among Enterobacteriaceae

\begin{tabular}{c}
\hline Organism $(\mathbf{n = 2 5 0 )}$ \\
\hline E.coli \\
\hline Klebsiella spp \\
\hline Enterobacter spp \\
\hline Proteus spp \\
\hline Citrobacter \\
\hline Serratia \\
\hline Morganella \\
\hline
\end{tabular}
AmpC Positive $(\mathbf{n}=\mathbf{2 8})$

\begin{tabular}{|c|}
\hline $6(21.4 \%)$ \\
\hline $15(53.6 \%)$ \\
\hline $4(14.3 \%)$ \\
\hline $0(0 \%)$ \\
\hline $3(10.7 \%)$ \\
\hline $0(0 \%)$ \\
$0(0 \%)$ \\
\hline
\end{tabular}

Resistance to Tobramycin, amoxicillin / Clavulanate and Amikacin were noted in between $30 \%$ and $19.6 \%$ of isolates. Only $2(1.2 \%)$ strains showed resistance to Imipenem. In the similar study conducted by Rong Wang et al., showed that $70.1 \%$ were resistant to
Ampicillin, $44.2 \%$ were resistant to Cefuroxime, $31.2 \%$ were resistant to Ceftazidime, $20.8 \%$ were resistant to Cefepime, and $75.3 \%$ were resistant to Cefoxitin (Rong WANG, 2009). 
Detection of AmpC beta-lactamases are based on a combination of sensitive screening and confirmation assays. Screening tests were done by the use of Double disc approximation test. And detection by Boronic acid disc test. In present study, Klebsiella represents 15 cases and E.coli 5 cases and Enterobacter 4 cases are detected as AmpC producers. Statistical analysis is done to test the association of distribution of AmpC on Enterobacteriaceae, the value obtained, Chi-square $=292.5$, $\mathrm{p}<0.0001$ and Fischer's Exact statistic $=64.11$, $\mathrm{p}<0.001$. This implies that there exist a significant difference between the distribution of AmpC on different Enterobacteriaceae.

A similar study conducted by Mona Wassef et al., shows Klebsiella spp represents 6 cases, 3 cases of E. coli and 2 cases of Enterobacter gave a positive Boronic acid Disc test. (Mona Waseef, 2013) But in the study of Shoorashetty et al., shows out of 200 isolates, 6 Klebsiella spp, 6 E. coli, 6 Enterobacter and 8 Citrobacter gave a positive Boronic acid Disc test (Shoorashetty, 2011). Similar study conducted by Shanthi et al., showed that, out of 77 isolates, 7 E.coli and 8 Klebsiella are gave a positive Boronic acid disc test for AmpC betalactamases.

$\beta$-lactam antibiotics are the most widely used chemotherapeutic agents. Commonest cause of resistant towards $\beta$-lactam antibiotics is the production of $\beta$-lactamases.

$\beta$-lactamases producers are an increasing cause of concern in hospitals as they produce a therapeutic confusion for the treating physician. In order to provide useful information for effective control and clinical therapy of infection, the resistant status and the rate of carrying AmpC $\beta$-lactamases of Enterobacteriaceae were investigated by phenotypic methods.

The study was conducted in a tertiary care hospital in Kerala, about 250 Enterobacteriaceae isolates were checked by Boronic Acid disc test to detect the resistance due to AmpC $\beta$-lactamases production in Enterobacteriaceae.

In the present study, first we concluded that from total Enterobacteriaceae isolates, the most commonly isolated organism was E.coli followed by Klebsiella spp. An overall antibiotic resistance pattern of the isolates showed high degree of resistance towards Ampicillin, Piperacillin, Amoxyclav and Third generation Cephalosporins and Fourth generation Cephalsporins. Whereas Imipenem, Meropenem, Colistin, Cefoperazone/Sulbactam, Piperacillin/ Tazobactum and Aminoglycosides were showing less resistance.

Amp C $\beta$-lactamase resistance was $11.2 \%$ (28/250) of circulating Enterobactericiae in the hospital set up. Knowledge of the presence of Amp C is critical especially in patients with sepsis where in vivo in vitro sensitivity difference will be there for higher generation cephalosporins.

Characterization, Identification of the antibiotic susceptibility patterns and detection of AmpC $\beta$-lactamases aid in Hospital infection control and help the physician to prescribe the most appropriate antibiotics, thus decreasing the selective pressure, which generates antibiotic resistance.

\section{How to cite this article:}

Ashish Jitendranath, Vishnupriya Anoobis, Geetha Bhai, Ivy Vishwamohanan and Ramani Bai, J.T. 2018. Occurrence and Detection of AmpC $\beta$-Lactamases among Enterobacteriaceae in a Tertiary Care Centre in Trivandrum, India. Int.J.Curr.Microbiol.App.Sci. 7(08): 176-181. doi: https://doi.org/10.20546/ijcmas.2018.708.023 